\title{
Relationship between the metabolic and lipid profile in follicular fluid of women undergoing in vitro fertilization
}

\author{
Simone Luti $^{1}$ | Tania Fiaschi $^{1}$ | Francesca Magherini ${ }^{1}$ | Pietro A. Modesti ${ }^{2}$ | \\ Paola Piomboni $^{3}$ | Laura Governini ${ }^{3}$ | Alice Luddi ${ }^{3}$ | Angela Amoresano ${ }^{4}$ | \\ Anna Illiano $^{4}$ | Gabriella Pinto ${ }^{4}$ | Alessandra Modesti ${ }^{1}$ (c) | Tania Gamberi ${ }^{1}$
}

${ }^{1}$ Department of Experimental and Clinical Biomedical Sciences "Mario Serio", University of Florence, Florence, Italy

${ }^{2}$ Department of Experimental and Clinical Medicine, University of Florence, Florence, Italy

${ }^{3}$ Department of Molecular and Developmental Medicine, University of Siena, Siena, Italy

${ }^{4}$ Department of Chemical Sciences, University of Naples Federico II, Naples, Italy

Correspondence

Alessandra Modesti, Department of

Experimental and Clinical Biomedical Sciences "Mario Serio", University of Florence, Viale G Morgagni, 50, Florence 50134, Italy.

Email: alessandra.modesti@unifi.it

\begin{abstract}
Among the follicular fluid (FF) components promoting the development of the oocyte are included glycoproteins, several fatty acids, and steroid hormones synthesized by the dominant follicle. For this, the analysis of the metabolites present in FF can determine the quality of the oocyte. FF composition is in part determined by local follicular metabolic processes and in part a plasma transudate. Since the causes of impaired fertility may be due to a metabolic imbalance, metabolomics is useful to identify low molecular weight metabolites. Oxidative stress is involved in human infertility and the use of metabolomics can be crucial to identify which other metabolites besides reactive oxygen species are involved in oxidative stress correlated to infertility. To obtain new information on the study of signaling molecules in FF, the knowledge of the lipid content will be important to improve information on the understanding of follicular development. The objective of this study is to identify (a) a metabolic profile and a lipid profile of FF in women undergoing in vitro fertilization and (b) to correlate the previous information obtained regarding adiponectin and oxidative stress with the metabolic and lipid profile obtained in the present study. As result, we found an increase in oxidative stress due to both an increase of androgens and an accumulation of lipids in the follicular environment and we suggest that this might be one of the causes of reduced fertility.
\end{abstract}

\section{KEYWORDS}

follicular microenvironment, lipidic profile, metabolic profile, network pathways

\section{1 | INTRODUCTION}

Follicular fluid (FF) is the product of the transudate from plasma and by the theca and the granulosa cells; during the development of the follicle, its composition changes in both quantity and quality. The interest of the researchers was, therefore, concentrated on the study of the follicular microenvironment composition to define the molecules involved in the several stages of follicular development. Among the FF components promoting the development and involved in the metabolism of the oocyte are included glycoproteins and steroid hormones synthesized by the dominant follicle. In the FF composition, there are several fatty acids and among them, it has been found linoleic acid in high quantities. For these reasons, it is easily understandable that the analysis of the metabolites present in 
this biological fluid can determine the quality of the oocyte (Freitas et al., 2017). Furthermore, to understand the causes of female infertility it becomes of enormous importance to study the composition of FF that may be used as a parameter to evaluate oocyte quality. Moreover, to fully understand if the FFs composition is only produced by antral cells or if it is in part determined by plasma transudate, we compared the composition of these two biological fluids in the same patients.

Since the causes of impaired fertility may be due to a metabolic imbalance, metabolomics can be used in reproductive medicine to identify and quantify low molecular weight metabolites present in the FF. This technique is able to identify molecules that are found downstream of gene expression and provides crucial information on cellular function (Bracewell-Milnes et al., 2017). It has been reported that oxidative stress is involved in human infertility. For this reason, the use of metabolomics can be crucial to identify which other metabolites besides reactive oxygen species (ROS) are involved in oxidative stress correlated to infertility (Olszak-Wąsik, BednarskaCzerwińska, Olejek, \& Tukiendorf, 2019). Recently, several studies have been conducted to identify small molecules in this biological fluid and some of these analyzed global metabolic profiles to determine the quality of follicular development.

To obtain new and in-depth information on the study of signaling molecules in FF, the knowledge of the lipid content can be crucial to improve information on the understanding of follicular development. Lipidomics using the power of mass spectrometry (MS), has recently highlighted the importance of studying lipids in FF as signaling molecules and to determine the lipid profile in the human ovary to elucidate the relationship between lipids and follicular development (Montani et al., 2019). In a previous paper Luti et al (accepted for publication) we investigated the level of the reactive oxygen metabolites diacron reactive oxygen metabolites ( $\mathrm{dROM}$ ) and the biological antioxidant potential biological antioxidant potential (BAP) correlated with adiponectin in FF of women who undergone in vitro fertilization (IVF), since both parameters, adiponectin, and oxidative stress, are involved in female infertility. With an increase in the concentration of ROS in FFs, is observed a decrease in fertility. Moreover, an accumulation of ROS in FF also leads to an increase in chronic inflammation which further causes a negative effect on fertility. Some cytokines are correlated with inflammation and in our previous results, we found a positive correlation between adiponectin and reactive oxygen metabolites in FF. We also highlighted the expression of this specific adipokine in granulosa and cumulus cells. We demonstrated that, in our analysis, the levels of adiponectin in FF are strongly correlated with 11-deoxicorticosterone and corticosterone precursors of mineral corticoids and cortisol and we found high levels of pregnenolone and progesterone suggesting the important role for the ovarian renin-angiotensin system in the oocyte development (Sneeringer, Penzias, Barrett, \& Usheva, 2011). The objective of the present study is to identify a metabolic profile of FF in women undergoing in vitro fertilization and a lipid profile in the same patients and in the same biological samples. Moreover, to further examine the data obtained from the metabolic profile, we built a network that connects the experimental compounds with the related genes/enzymes/metabolic pathways. In the end, we correlated the information obtained in our previous study with the metabolic and lipid profile obtained in the present study.

In the end, we compared the FF metabolic and lipid profile with the serum in the same patients to better understand how the composition of the follicular microenvironment is influenced by the composition of the plasma and how much is the product of the transudate of the endocrine cells in the ovary surrounding the follicle.

\section{2 | RESULTS}

\section{1 | Metabolomic analysis using GC-MS}

We characterized the metabolic profile of FF and we compared it with the metabolic profile of sera from healthy women undergoing IVF, through GC-MS as reported in methods. FF composition is in part determined by local follicular metabolic processes and in part by plasma transudate. The follicle wall acts as a coarse molecular sieve, allowing small metabolites to pass through while restricting the access of molecules $>100 \mathrm{kDa}$. For this reason, it is important to analyze, in the same women, serum and FF composition and compare the results obtained. We performed our metabolomic analysis using a semitargeted approach (Liu \& Locasale, 2017) that allow the unambiguously identification and quantifications of molecules. However, this approach leads to a smaller number of identified molecules in comparison to untargeted methods but it allows a correct and unambiguous quantification. The identification of each compound was obtained through Fiehn library 2013 that is composed by 1,447 compounds and quantification is obtained by the interpolation of extracted ion chromatogram peak area onto the relative calibration (Lorito et al., 2020). Our methods allow to identify 56 compounds, among them 22 are present in both biological samples, 11 were present only in serum and 21 in FF as reported in Table 1. Several of the identified compounds are involved in the main metabolic pathways such as sugars metabolism, amino acid metabolism, nitrogen metabolism, and tricloroacetic acid cycle ( $p<.05$ with MetaboAnalyst).

Among the 22 identified in both sera and FFs, 12 of them, showed no significant differences between the two biological samples; on the contrary, 10 of them have a statistically different concentration between serum and FF. In particular, in serum samples 1,5-anhydro-D-sorbitol shows a statistically significant increase $(p<.01)$ of about $46 \%$ in comparison to FF. The same trend is shown for 2-amino-2-methyl-1,3-propanediol, L-ornithine, L-valine, acetohydroxamic acid, and citric acid which concentration show an increase in serum of 2.5 ( $p<.001), 1.8$ ( $p<.05), 2.5$ ( $p<.001), 5.0(p<.01)$, and $1.5(p<.001)$ fold compared to FF; the results are reported in Figure 1a. All these compounds are involved in sugar and amino acid metabolism and the urea cycle. In contrast, lactate, malonate, and pyruvate, involved in glucose metabolism, show an opposite trend, since they are $3.0(p<.01), 4.0(p<.001)$, and $2.5(p<.05)$ fold more abundant in FF than in serum. Moreover, the amino acid glycine 
TABLE 1 List of Metabolites identified in serum and follicular fluid by gas chromatography-mass spectrometry (GC-MS) analysis

\begin{tabular}{|c|c|c|c|c|}
\hline \multicolumn{2}{|c|}{ Metabolite name } & Retention time & $\begin{array}{l}\text { CAS } \\
\text { number }^{a}\end{array}$ & KEGG ID ${ }^{b}$ \\
\hline \multicolumn{5}{|c|}{ Metabolites in common between serum and follicular fluid } \\
\hline 1 & 1,5-Aanhydro-D-sorbitol & [16.967] & $154-58-5$ & $\mathrm{C} 07326$ \\
\hline 2 & 2-Aamino-2-methyl-1,3-propanediol & {$[10.56]$} & $115-69-5$ & C11260 \\
\hline 3 & D-Sorbitol & [17.898] & $50-70-4$ & C00794 \\
\hline 4 & L-(+) Lactic acid & [6.851] & 79-33-4 & C00186 \\
\hline 5 & L-Ornithine & [16.632] & $70-26-8$ & $\mathrm{C} 00077$ \\
\hline 6 & L-Threonine & [11.464] & $72-19-5$ & $\mathrm{C} 00188$ \\
\hline 7 & L-Valine & [9.151] & $72-18-4$ & $\mathrm{C} 00183$ \\
\hline 8 & Acetohydroxamic acid & {$[7.72]$} & $546-88-3$ & $\mathrm{C06808}$ \\
\hline 9 & Alpha ketoglutaric acid & [13.859] & $328-50-7$ & $\mathrm{C} 00026$ \\
\hline 10 & Citric acid & [16.615] & $5949-29-1$ & $\mathrm{C} 00158$ \\
\hline 11 & Ethanolamine & [9.879] & $141-43-5$ & C00189 \\
\hline 12 & Glyceric acid & {$[10.735]$} & $473-81-4$ & $\mathrm{C} 00258$ \\
\hline 13 & Glycine & {$[10.456]$} & $56-40-6$ & $\mathrm{C} 00037$ \\
\hline 14 & Isopropyl beta-D-1-thiogalactopyranoside & [19.097] & $367-93-1$ & $\begin{array}{l}\mathrm{C} 03619 \\
\mathrm{C} 02327\end{array}$ \\
\hline 15 & Malonic acid & [8.919] & $141-82-2$ & $\mathrm{C00383}$ \\
\hline 16 & Oxalic acid & [7.883] & $144-62-7$ & C00209 \\
\hline 17 & Palmitic acid & [18.846] & $64519-82-0$ & $\mathrm{C} 00249$ \\
\hline 18 & Phosphoric acid & [9.966] & 7664-38-2 & C00009 \\
\hline 19 & Pyruvic acid & [6.714] & $127-17-3$ & $\mathrm{C} 00022$ \\
\hline 20 & Threonic acid & [13.652] & 7306-96-9 & C01620 \\
\hline 21 & Urea & [9.599] & $57-13-6$ & C00086 \\
\hline 22 & Uric acid & [19.331] & $66-22-8$ & $\mathrm{C} 00366$ \\
\hline \multicolumn{5}{|c|}{ Metabolites only in serum } \\
\hline 23 & 1-Octadecene & [16.36] & $112-88-9$ & \\
\hline 24 & $\begin{array}{l}\text { 12a-Hydroxy-9-demethylmunduserone- } \\
\text { 8-carboxylic acid }\end{array}$ & [26.831] & $0-00-0$ & C16189 \\
\hline 25 & 2-Hydroxypyridine & [6.519] & $142-08-5$ & $\mathrm{CO} 2502$ \\
\hline 26 & 3-Phenyllactic acid & [13.981] & $828-01-3$ & C01207 \\
\hline 27 & L-Glutamic acid & [13.338] & $56-86-0$ & $\mathrm{C} 00025$ \\
\hline 28 & L-Methionine & [11.835] & $63-68-3$ & $\mathrm{C} 00073$ \\
\hline 29 & N-Acetyl-D-tryptophan & [21.742] & $0-00-0$ & C03137 \\
\hline 30 & Aspartic acid & [13.207] & $56-84-8$ & $\mathrm{C} 00049$ \\
\hline 31 & Elaidic acid & [20.508] & $112-79-8$ & $\mathrm{C} 01712$ \\
\hline 32 & Glycerol & [9.941] & $56-81-5$ & $\mathrm{C} 00116$ \\
\hline 33 & Myo-inositol & [19.354] & $87-89-8$ & $\mathrm{C} 00137$ \\
\hline 34 & Xanthotoxin & [20.715] & $298-81-7$ & C01864 \\
\hline \multicolumn{5}{|c|}{ Metabolites only in follicular fluid } \\
\hline 35 & 2-Butyne-1,4-diol & [9.446] & $110-65-6$ & $\mathrm{C} 02497$ \\
\hline 36 & 2-Hydroxybutyric acid & {$[7.852]$} & $565-70-8$ & $\mathrm{C} 05984$ \\
\hline 37 & 2-Ketoisocaproic acid & [9.041] & $4502-00-5$ & $\mathrm{C03467}$ \\
\hline 39 & D (+)Altrose & [17.46] & & $\mathrm{C} 06464$ \\
\hline 40 & D-Glucose & [17.426] & $59-23-4$ & $\mathrm{C} 00031$ \\
\hline 41 & L-Alanine & {$[7.4748]$} & $56-41-7$ & C00041 \\
\hline 42 & L-Glutamic acid & [13.232] & $56-86-0$ & $\mathrm{C} 00025$ \\
\hline 44 & L-Glutamine & [14.083] & $56-85-9$ & \\
\hline 45 & L-Proline & [10.321] & $147-85-3$ & $\mathrm{C} 00148$ \\
\hline 46 & L-Pyroglutamic acid & [13.218] & $98-79-3$ & C01879 \\
\hline 47 & L-Serine & [11.174] & $56-45-1$ & $\mathrm{C} 00065$ \\
\hline 48 & L-Valine & [9.151] & $72-18-4$ & C00183 \\
\hline 49 & N-Methylglutamic acid & [12.426] & $6753-63-4$ & C01046 \\
\hline
\end{tabular}

(Continues) 
TABLE 1 (Continued)

\begin{tabular}{lllll} 
Metabolite name & & \multicolumn{2}{l}{ CAS } \\
& Retention time & number $^{\mathrm{a}}$ & KEGG ID $^{\mathrm{b}}$ \\
\hline 50 & Adipamide & {$[15.975]$} & $628-94-4$ & \\
51 & Diglycerol & {$[15.958]$} & $627-82-7$ & \\
52 & Glycerol 1-phosphate & {$[16.056]$} & $34363-28-5$ & C03189 \\
53 & Glycolic acid & {$[7.049]$} & $79-14-1$ & C00160 \\
54 & Hexanoic acid & {$[7.11]$} & $142-62-1$ & C01585 \\
55 & Methyl-beta-D-galactopyranoside & {$[16.935]$} & $1824-94-8$ & C03619 \\
56 & Stearic acid & {$[20.675]$} & $57-11-4$ & C01530 \\
\hline
\end{tabular}

${ }^{a}$ Chemical Abstract Service number.

${ }^{\mathrm{b}}$ KEGG identifier (https://www.genome.jp/kegg/).

shows in FF an increase of 1.5 fold $(p<.05)$ in comparison with serum as shown in Figure 1b.

In addition, in our analyses, we identified some compounds detectable only in FF and not in serum and vice versa.

The metabolites identified only in FF, glycolate, serine, and glutamine, are mainly involved in glyoxylate and dicarboxylate metabolism ( $p<.05$ with MetaboAnalyst) or in amino acids metabolism.

\section{2 | Lipidomics analysis using MALDI-TOF MS}

In an effort to better investigate the metabolic profile and the relationship between different metabolic species in FFs and sera of women undergoing IVF, a lipidomic approach was performed by using MALDI-TOF MS.

MALDI MS analyses were performed in FFs and sera methanol extracts as reported in methods. Comparison between the MALDI spectra recorded for sera from women $<35$ and $>35$ years old is reported in Figure 2 where a different peak distribution underlined dissimilar metabolic profile in two spectra ranging from 500 to
$1100 \mathrm{~m} / \mathrm{z}$ values is shown. To investigate the different species detected by MALDI analysis, MSMS spectra were run by selecting the peaks exhibiting the better signal to noise ratio. As a whole, the interpretation and the assignment of the different species registered within on the MALDI TOF spectra was performed both by fragmentation spectra of the most intense peak obtained by MALDI TOF analysis and by comparing the experimental data to lipid databases available on the web. In sera, were identified different classes of compounds playing crucial roles within the cell, namely phosphatidylcholines (PCs), phosphatidylethanolamines (PEs), phosphatidylinositols (PIs) and phosphatidylserines. These compounds represent the main constituents of membranes and organelles, play a crucial role in cell signaling, and over and above have a nutritional function. The identified species are summarized in Supporting Information data (Tables 1S-2S, respectively, for sera and FF). Several authors reviewed the relevance of ion acquisition mode even in combination with different used matrices (Leopold, Popkova, Engel, \& Schiller, 2018). Most of them deal with a negative ion mode especially for triacylglycerols, fatty acids, and some phospholipids. In this study, the combination of the extraction steps to the use of positive ion mode and DHB (2,5-Dihydroxybenzoic acid) matrix was designed to (a)

High serum metabolites

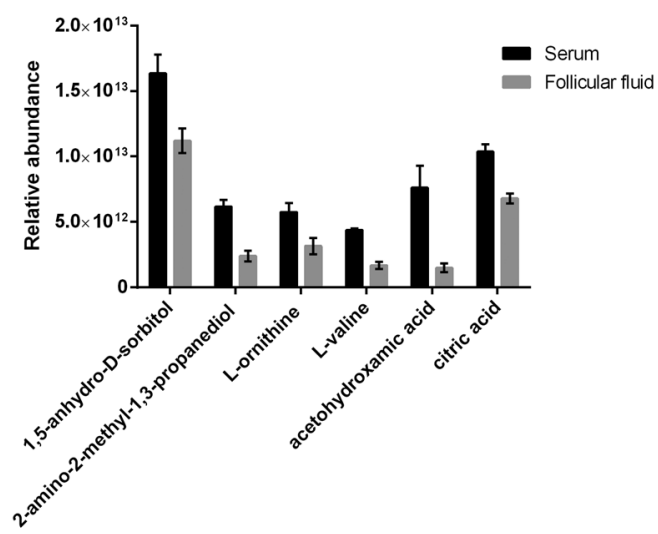

(b)

High FF metabolites

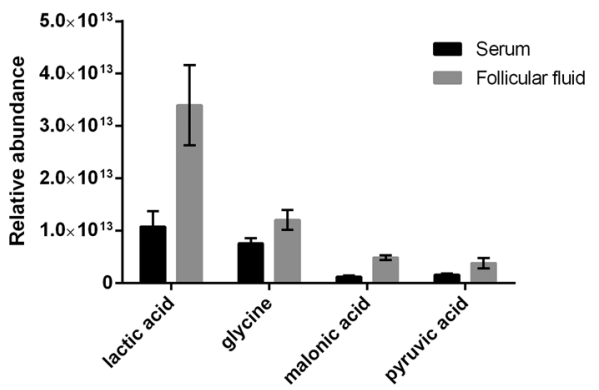

FIGURE 1 Metabolomic analysis using GC-MS. Metabolites in common between serum and follicular fluids (FF). (a) Histogram representation of metabolites whose relative abundance is higher in serum. (b) Histogram representation of metabolites whose relative abundance is higher in FF 
(a)
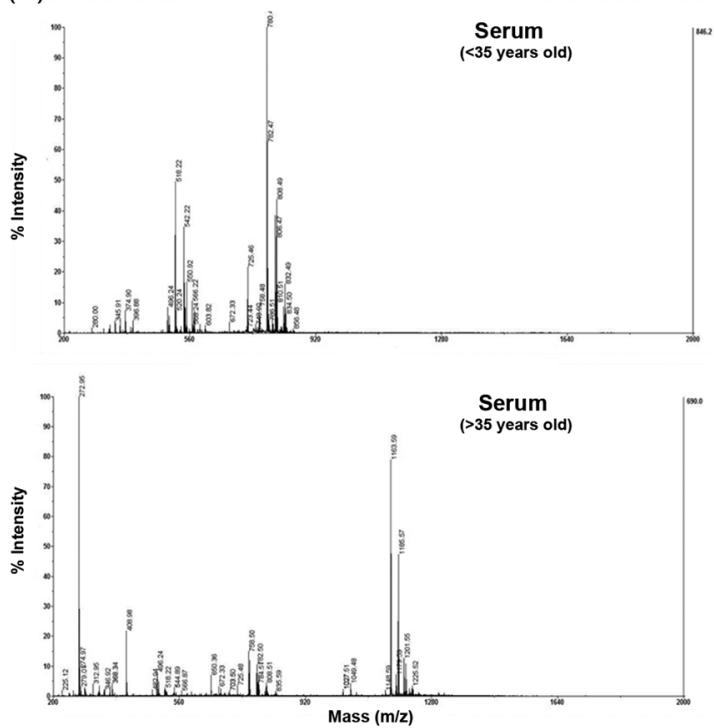

(b)

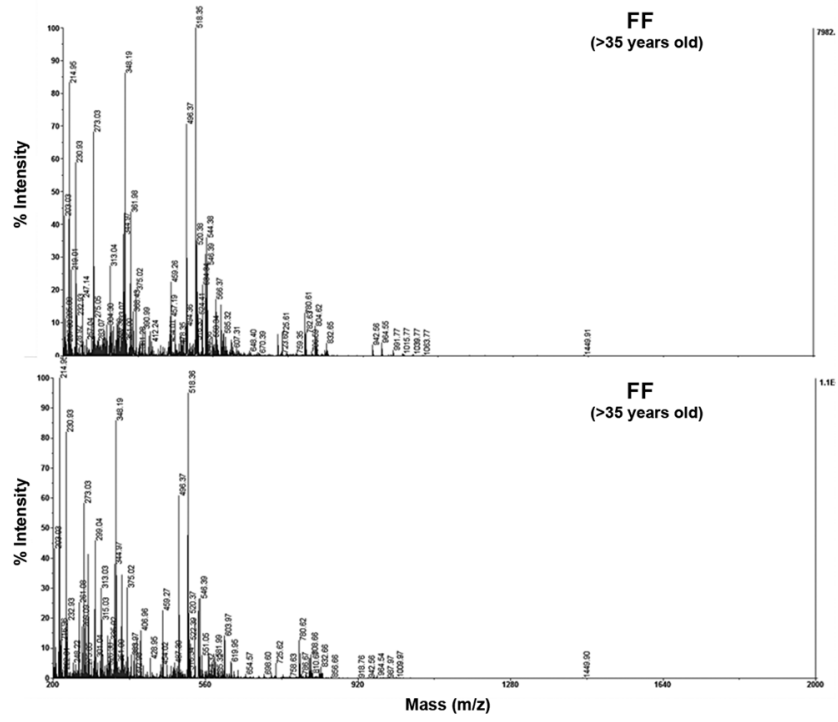

FIGURE 2 Lipidomic analysis by MALDI-TOF mass spectrometry. MALDI spectra of serum pool (a) and FF pool (b) in the range 200-2000 Da. MALDI-TOF, matrix-assisted laser desorption/ionization time-of-flight

investigate mainly the profile of phospholipids. The nitrogen centered cations or phosphate anions of the polar head of PC or PE make these molecules easy ionizable in both modes during the MALDI process (Berry et al., 2011). These molecules were also the most abundant ion species detected in our MALDI spectra acquired in positive mode for both biofluids.

To minimize the individual biological variability, samples were pooled according to the donor's age (pool $<35$ and pool $>35$, years old) and treated as medium samples. Thus, mass spec analysis was performed on the two-pooled samples and the MALDI spectra are reported in Figure 2a. Mass spectral analysis performed on pool samples revealed a peak distribution in perfect agreement with the analyses carried out on the single ones Analogously to the sera, the FFs were treated and aliquots of methanolic extracts were analyzed by MALDI-TOF. MALDI MS analyses performed on the two different classes of biological fluids revealed that methanol extracts from FFs showed a different peak distribution with respect to serum samples as shown in Figure 2b. In particular, an inversion of signal intensities in the regions of the spectrum centered on 500 and $900 \mathrm{Da}$ is observed. As a whole, these analyses could represent a useful tool to obtain a rapid and sensitive fingerprinting of biological fluids.

\section{3 | Metabolomic and lipidomic interaction network}

From the metabolites and lipids list obtained from sera and FFs analysis, we built interaction networks. We used MetScape (http:// metscape.med.umich.edu) an App available on the open-source software platform Cytoscape, which provides a bioinformatics framework for the visualization and interpretation of metabolomic data
(Karnovsky et al., 2012). It allows to analyze metabolic networks of genes, compounds, enzymes, and pathways using an internal database that integrates data from KEGG and EHMN (Edinburgh Human Metabolic Network; Ma et al., 2007). Metabolic networks can be divided into subgroups with the capability to visualize potential enhanced pathways in each group (Ma et al., 2019). Considering the compounds obtained with the metabolomic analysis, we built an interaction network with the compounds that showed significant differences between serum and FF reported in Table 2.

As evident from the comparison between Table 1 and 2, not all the metabolites identified by the Fiehn library were recognized by the MetScape platform.

The selected networks obtained show an involvement of the metabolism of the sulfur amino acids and other amino acids (glycine, serine, alanine, threonine, and lysine). Moreover, we noted an involvement of sugars metabolism (glycolysis and gluconeogenesis) and leukotriene metabolism as reported in detail in Figure 3.

The selected networks built by MetScape using the compounds identified only in FF indicate the involvement of purine and pyrimidine metabolism, the folate metabolism as indicate in Figure 4 and Table 3 as well as the metabolism of several amino acids such as histidine, arginine, proline, glutamate, aspartate, and asparagine. In addition, we found affected aminosugars metabolism and degradation of valine, leucine, and isoleucine. Moreover, we noted an involvement of sugars metabolism (fructose, mannose, and galactose metabolism) and lipid metabolism (de novo fatty acid biosynthesis; glycerophospholipid metabolism). The interaction network obtained with the compounds identified only in serum showed the involvement of metabolism of phospho and glycero-pospho lipids (Figure S1 of Supporting Information data). 
TABLE 2 List of different metabolites between serum and follicular fluid identified by GC-MS used to build MetScape network

\begin{tabular}{|c|c|c|c|}
\hline $\begin{array}{l}\text { Metabolite } \\
\text { number }\end{array}$ & Metabolite name & $\begin{array}{l}\text { CAS } \\
\text { number }^{b}\end{array}$ & KEGG ID \\
\hline \multicolumn{4}{|c|}{ List of metabolites used to build MetScape network ${ }^{a}$} \\
\hline 4 & L-(+) Lactic acid & 79-33-4 & C00186 \\
\hline 5 & L-Ornithine 2 & $70-26-8$ & C00077 \\
\hline 7 & L-Valine 1 & $72-18-4$ & $\mathrm{C} 00183$ \\
\hline 10 & Citric acid & $5949-29-1$ & $\mathrm{C} 00158$ \\
\hline 13 & Glycine & $56-40-6$ & $\mathrm{C} 00037$ \\
\hline 15 & Malonic acid 1 & $141-82-2$ & $\mathrm{C} 00383$ \\
\hline 19 & Pyruvic acid & $127-17-3$ & $\mathrm{C} 00022$ \\
\hline \multicolumn{4}{|c|}{ Pathways by MetScape platform ${ }^{\text {a }}$} \\
\hline \multicolumn{4}{|c|}{ Bile acid biosynthesis } \\
\hline \multicolumn{4}{|c|}{ Glycine, serine, alanine and threonine metabolism } \\
\hline \multicolumn{4}{|c|}{ Glycolysis and Gluconeogenesis } \\
\hline \multicolumn{4}{|c|}{ Leukotriene metabolism } \\
\hline \multicolumn{4}{|c|}{ Lysine metabolism } \\
\hline \multicolumn{4}{|c|}{ Methionine and cysteine metabolism } \\
\hline \multicolumn{4}{|c|}{ Porphyrin metabolism } \\
\hline \multicolumn{4}{|c|}{ TCA cycle } \\
\hline \multicolumn{4}{|c|}{$\begin{array}{l}\text { Urea cycle and metabolism of arginine, proline, glutamate, } \\
\text { aspartate, and asparagine }\end{array}$} \\
\hline \multicolumn{4}{|c|}{ Valine, leucine, and isoleucine degradation } \\
\hline \multicolumn{4}{|c|}{ Vitamin B9 (folate) metabolism } \\
\hline
\end{tabular}

Abbreviations: GC-MS, gas chromatography-mass spectrometry; TCA, tricloroacetic acid.

${ }^{\mathrm{a}}$ (http://metscape.med.umich.edu).

${ }^{\mathrm{b}}$ Chemical Abstract Service number.

${ }^{\mathrm{c}}$ KEGG identifier (https://www.genome.jp/kegg/).

Considering the networks built for FF using the lipid list, the subgroups obtained from the interaction analysis demonstrate enhanced metabolism of glycerophospholipid, as well as pantothenate and CoA biosynthesis (Figure 5). The subnetworks obtained from sera show enhanced metabolism of PI phosphate, and glycerophospholipid (Figure 2S of Supporting Information data).

\subsection{Correlation analysis between metabolites with both adiponectin and oxidative stress level}

Adiponectin controls the functions of somatic and germ cells through the activation of the hypothalamic-ipofisi-gonadal axis. It also plays an important role in the synthesis of steroid hormones by follicular cells promoting the development of the oocyte. Moreover, another factor influencing the oocyte maturation is the level of oxidative stress that we evaluated in a previous publication where we found a significative correlation among adiponectin, oxidative stress, and several steroids hormones in FF. Here, we determined in FF the Spearman's correlation between adiponectin and oxidative stress (BAP/dROM) with the various metabolites measured in this study and, regarding adiponectin, we found no correlation. On the contrary, a positive correlation is evident in Figure 6 between the BAP and hydroxybutirrate $(p=.001 ; r=.6418)$, glycerol 1 -phosphate $(p=.040$; $r=.4295)$ and glycolic acid ( $p=.025 ; r=.4647)$. In contrast, a negative correlation is evident with malonate $(p=.009 ; r=-.5319)$, hexanoic acid ( $p=.024 ; r=-.4697)$, and methyl-beta-D-galactopyranoside $(p=.039 ; r=-.4334)$. Correlating the reactive oxygen metabolites (dROM) with the metabolites, a positive correlation is observed with pyruvate $(p=.011 ; r=.5193)$, glycerol 1 -phosphate $(p=.038$; $r=.4351)$ and glycolic acid $(p=.043 ; r=.4244)$ as shown in Figure 7.

The results obtained in serum, (Figure S3 of Supporting Information data) point out a significant negative correlation between adiponectin with citrate $(p=.0178 ; r=-.5663)$ and phosphoric acid $(p=.0496 ; r=-.4828)$ and a positive correlation with glycine $(p=.0502 ; r=.4846)$. Moreover, we observed a significant positive correlation between BAP and malonate ( $p=.003 ; r=.6697)$; on the contrary, a negative correlation among BAP and urea $(p=.034$; $r=-.5160)$ and Myo-inositol $(p=.018 ; r=-.5664)$ is shown. With regard to the correlation between the levels of oxidative species (dROM) and the metabolites we observed a significantly negative Spermann correlation index among dROM, and citrate $(p=.009$; $r=-.6085)$, ethanolamine $(p=.010 ; r=-.6047)$ and glutamate $(p=.015 ; r=-.5802)$. We reported in the supplementary materials (Figure S3) the histograms concerning the correlation data obtained by analyzing the serum values to better focus on the follicle data.

\section{3 | DISCUSSION AND CONCLUSION}

The purpose of this study was the analysis of the follicular microenvironment in women undergoing in vitro fertilization. The study is based on a metabolomic analysis performed by gas chromatography coupled with MS and a lipidomic analysis by MALDI-TOF MS. The content evaluation is made by comparing the results obtained on the FF with those carried out on the serum of the same women.

Regarding the content of the metabolites, the FF samples showed high levels of lactate and pyruvate compared to the serum in line with published data demonstrating that growth of the follicle and production of steroid hormones are both dependent by the glucose metabolism (Boland, Humpherson, Leese, \& Gosden, 1993). In fact, the follicle mainly uses the glycolytic pathway to obtain energy for its development and steroidogenesis. In our study, we showed an increase in lactate concentration in FF composition compared to serum. This increase is justified because in ovarian hyperstimulation cycles there is an important increase in anaerobic metabolism (Gull et al., 1999). Harlow (Harlow, Winston, Margara, \& Hillier, 2010) reported that the increase in lactate concentration is due to an increase in glycolysis in granulosa cells. This increase is a consequence of the greater energy demands during the development of the follicle and a consequent lower availability of oxygen (Sutton-McDowall, Gilchrist, \& Thompson, 2010). We also highlighted an increase in the level of pyruvic acid in FF compared to the serum that confirms how the FF provides an anaerobic energy metabolism for the maturation 


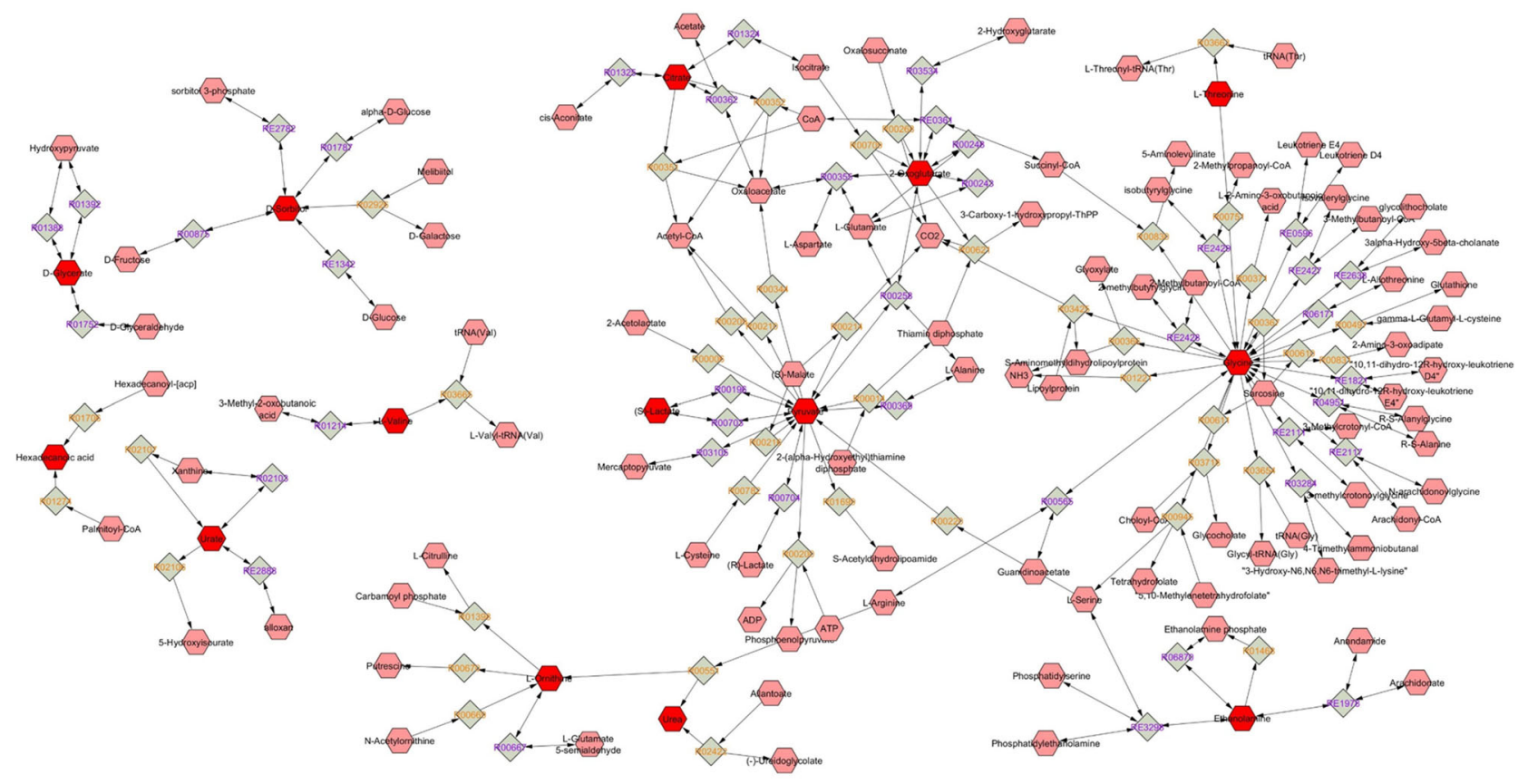

FIGURE 3 Metabolomic Network interaction analysis on serum and FF metabolites. Selected Network interaction analysis of the differentially abundant metabolites between serum and follicular fluid identified by GC-MS. The network analysis was carried out by using the MetScape 3 App for Cytoscape (http://metscape.med.umich.edu). FF, follicular fluid; GC-MS, gas chromatography/mass spectrometry [Color figure can be viewed at wileyonlinelibrary.com]

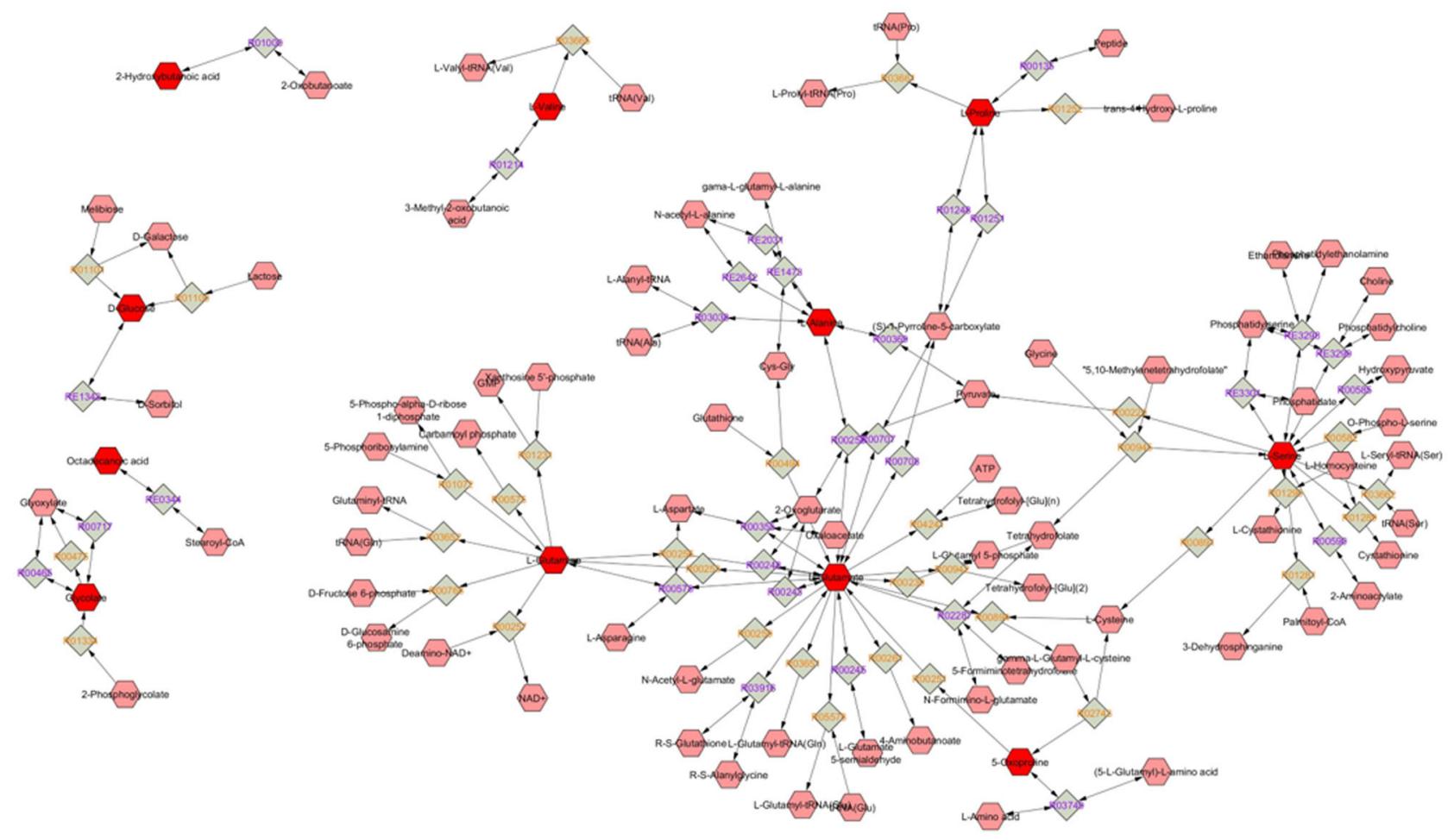

FIGURE 4 Metabolomic Network interaction analysis on follicular fluid metabolites. Selected Network interaction analysis of the metabolites identified only in follicular fluid by GC-MS. The network analysis was carried out by using the MetScape 3 App for Cytoscape (http://metscape.med.umich.edu). GC-MS, gas chromatography/mass spectrometry [Color figure can be viewed at wileyonlinelibrary.com] 
TABLE 3 List of metabolites identified in follicular fluid identified by GC-MS used to build MetScape network

\begin{tabular}{|c|c|c|c|}
\hline $\begin{array}{l}\text { Metabolite } \\
\text { number }\end{array}$ & Metabolite name & $\begin{array}{l}\text { CAS } \\
\text { number }\end{array}$ & KEGG ID ${ }^{c}$ \\
\hline \multicolumn{4}{|c|}{ List of metabolites used to build MetScape network ${ }^{a}$} \\
\hline 36 & 2-Hydroxybutyric acid & $565-70-8$ & C05984 \\
\hline 40 & D-Glucose 1 & $59-23-4$ & $\mathrm{C} 00031$ \\
\hline 41 & L-Alanine 1 & $56-41-7$ & $\mathrm{C} 00041$ \\
\hline 42 & L-Glutamic acid 3 & $56-86-0$ & $\mathrm{C} 00025$ \\
\hline 43 & L-Glutamine 1 & $56-85-9$ & $\mathrm{C} 00064$ \\
\hline 45 & L-Proline 2 & $147-85-3$ & $\mathrm{C} 00148$ \\
\hline 47 & L-Serine 2 & $56-45-1$ & $\mathrm{C} 00065$ \\
\hline 48 & L-Valine 2 & $72-18-4$ & $\mathrm{C} 00183$ \\
\hline \multicolumn{4}{|c|}{ Pathways by MetScape platform ${ }^{a}$} \\
\hline \multicolumn{4}{|c|}{ Aminosugars metabolism } \\
\hline \multicolumn{4}{|c|}{ De novo fatty acid biosynthesis } \\
\hline \multicolumn{4}{|c|}{ Fructose and mannose metabolism } \\
\hline \multicolumn{4}{|c|}{ Galactose metabolism } \\
\hline \multicolumn{4}{|c|}{ Glycerophospholipid metabolism } \\
\hline \multicolumn{4}{|c|}{ Glycine, serine, alanine and threonine metabolism } \\
\hline \multicolumn{4}{|c|}{ Glycosphingolipid metabolism } \\
\hline \multicolumn{4}{|c|}{ Histidine metabolism } \\
\hline \multicolumn{4}{|c|}{ Methionine and cysteine metabolism } \\
\hline \multicolumn{4}{|c|}{ Purine metabolism } \\
\hline \multicolumn{4}{|c|}{ Aminosugars metabolism } \\
\hline \multicolumn{4}{|c|}{ Pyrimidine metabolism } \\
\hline \multicolumn{4}{|c|}{$\begin{array}{l}\text { Urea cycle and metabolism of arginine, proline, glutamate, aspartate } \\
\text { and asparagine }\end{array}$} \\
\hline \multicolumn{4}{|c|}{ Valine, leucine, and isoleucine degradation } \\
\hline \multicolumn{4}{|c|}{ Vitamin B3 (nicotinate and nicotinamide) metabolism } \\
\hline Vitamin $\mathrm{E}$ & folate) metabolism & & \\
\hline
\end{tabular}

Abbreviation: GC-MS, gas chromatography-mass spectrometry.

a (http://metscape.med.umich.edu).

${ }^{\mathrm{b} C h e m i c a l}$ Abstract Service number.

${ }^{c}$ KEGG identifier (https://www.genome.jp/kegg/).

and development of the oocytes (Piñero-Sagredo, Nunes, de Los Santos, Celda, \& Esteve, 2010). In our study, we identified higher serum levels compared to FF of the amino acid valine. It is known from the literature that the essential amino acid valine is significantly higher in the serum in women with repeated failure of in vitro fertilization (Xia et al., 2014). In addition, among the metabolites present only in FF we have identified the presence of amino acids such as alanine, glutamic acid, glutamine, proline, and serine. From the literature, it is known that the amino acid serine has a high concentration in patients with ovulatory dysfunction (Zhao et al., 2012). In addition, Zang reports that in women with Polycystic ovary syndrome (PCOS) associated with insulin resistance there was an increase in the FF of glutamic acid levels while high levels of aspartic acid and serine were associated with successful pregnancy (Zhang et al., 2014). In our study, we built a network using MetScape that indicates the involvement of leukotriene metabolism using the list of metabolites showing statistically differences between FF and serum.
It is known that leukotrienes, prostaglandins, and probably other eicosanoids play a fundamental role in the ovulation process. In fact, they are involved in the rupture mechanism of the follicular wall. Indeed, it seems likely that the altered metabolism of these metabolites is responsible for poor female fertility in humans (Niringiyumukiza, Cai, \& Xiang, 2018). Moreover, we found the involvement of purine and pyrimidine metabolism in FF. Although at least to our knowledge no results are known on the role of purine and pyrimidine metabolism in human reproduction, Chen et al recently identified that purine metabolism and the increase in adenine concentration in FF may compromise the development of oocyte in sows (Chen et al., 2019). Analyzing the networks obtained we noticed the involvement of many pathways related to lipid metabolism in FF and it is known from the literature that an intravenous infusion of lipids induces the production of androgens at the follicle level (Mai et al., 2008). Gervais, Battista, Carranza-Mamane, Lavoie, and Baillargeon (2015) suggest that the local production of androgens is associated with the presence of intraovarian lipids and that this association can lead to inflammatory phenomena implicated in intraovarian androgenesis, which, for example, characterizes women with PCOS. In our previous study, we reported in FF high levels of steroid precursors of mineralocorticoid hormones in comparison to serum and these can be involved in the ovarian renin-angiotensin system that influences the development of the follicle. Analyzing the lipid list obtained we observed that the most abundant peak in serum was assigned to the sodiated form of phosphatidylcholine 34:2 (PC$34: 2-780.5 \mathrm{~m} / \mathrm{z}$ ) confirmed by the MS/MS spectrum and previously reported by others (Berry et al., 2011). Moreover, the corresponding lysophosphatidylcholine was less abundant than the PC in pooled sera. This finding may be due to the higher activity of phoshpolypases A2 (PLA2) releasing the fatty acids from lipids. Several authors investigated a strong correlation between PLA2 activity and the oocyte quality supporting oocyte failure to form pronuclei (Cordeiro et al., 2018).

Furthermore, analyzing the networks built with the lipid list in FF, we noticed enhanced metabolism of glycerophospholipid, and pantothenate and CoA biosynthesis involved in the beta-oxidation of fatty acids. This involvement can lead to incomplete oxidation with the accumulation of lipid metabolites with consequent lipotoxicity in the ovaries (Gonzalez, Lane, Knight, \& Robker, 2018). Jungheim et al. (2011) has noted that this induces the production of low-quality oocytes during in vitro fertilization. Mice fed on diets rich in fatty acids show low levels of ovulation and fertilization and an increase in the apoptosis of granulosa and cumulo cells (Wu et al., 2010). We found a positive correlation in FF between the level of biological antioxidant potential/oxidative species (BAP and dROM) previously determined and two significant metabolites, glycerol 1-phosphate and glycolic acid, related to mitochondrial function. Glycerol 1-phosphate is the product of the catabolism of triglyceride and its dismetabolism could lead to higher glycerol and triglyceride levels and could be a source of reactive oxygen species involved in the development of obesity and metabolic syndrome, impairing the fertility (Dludla et al., 2018). Glycolic acid is a major precursor of oxalate 

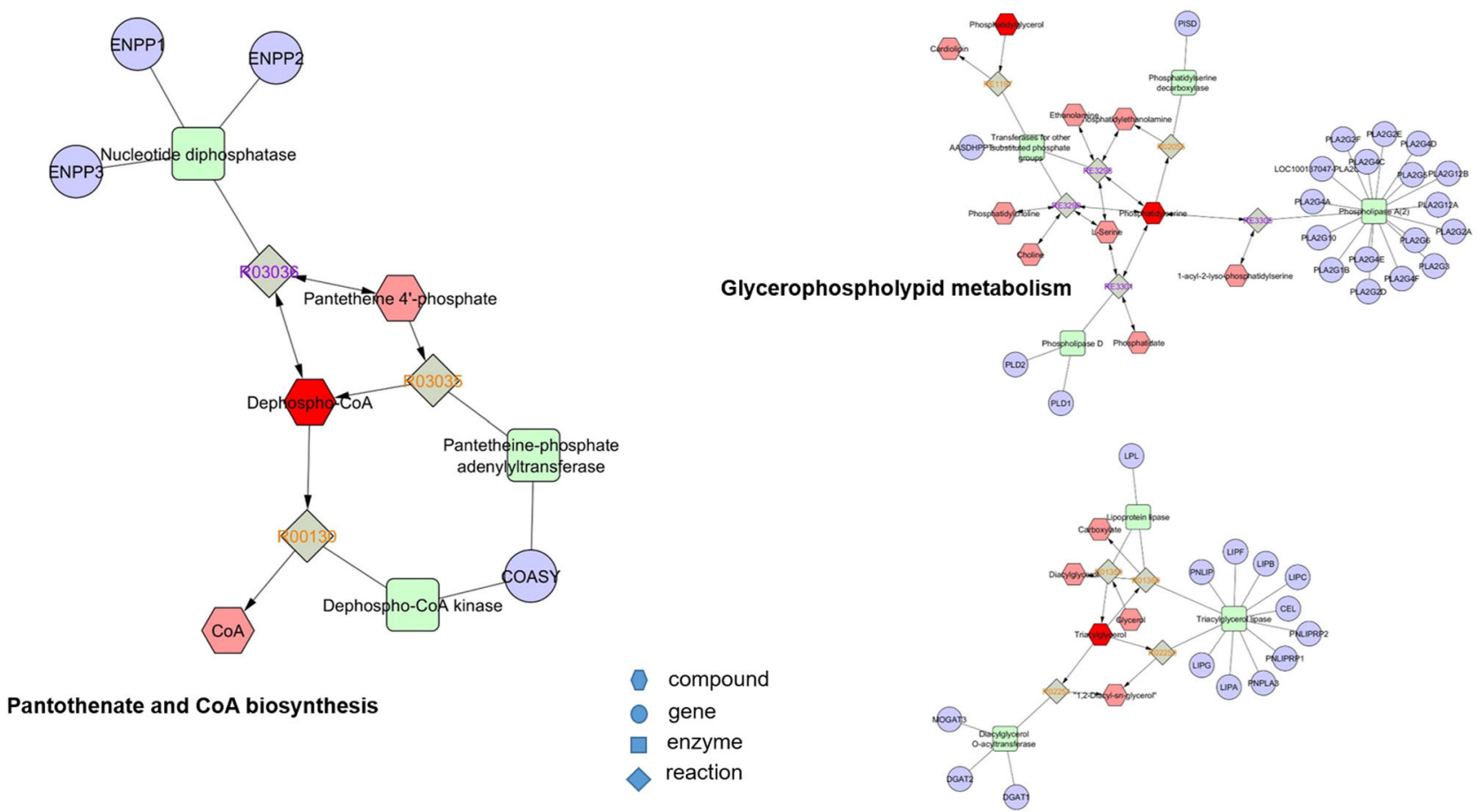

FIGURE 5 Lipidomic Network interaction analysis on follicular fluid metabolites. Selected subnetwork interaction analysis of lipids, genes, and compounds identified only in follicular fluid by Maldi-TOF mass spectrometer analysis. The network analysis was carried out by using the MetScape 3 App for Cytoscape (http://metscape.med.umich.edu). TOF, time-of-flight [Color figure can be viewed at wileyonlinelibrary.com]
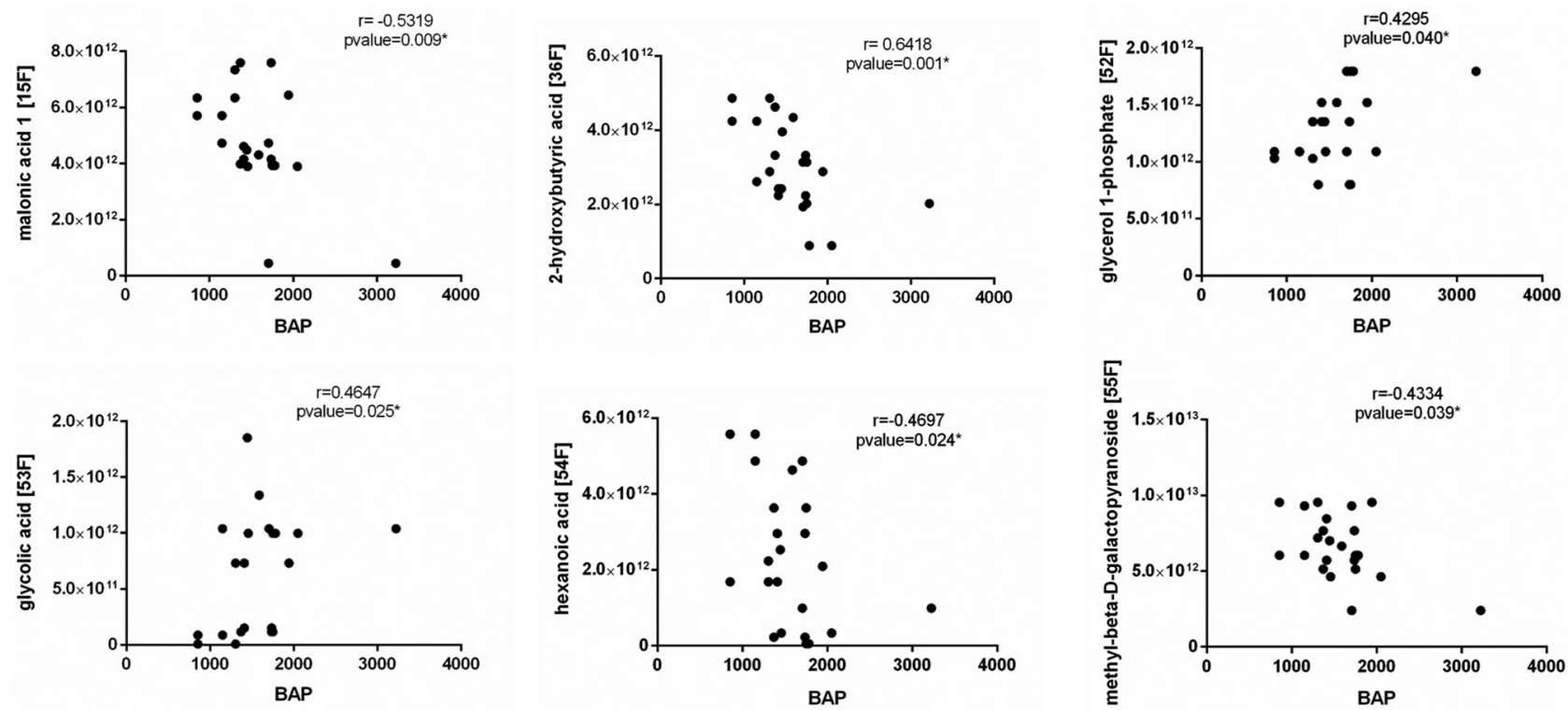

FIGURE 6 Spearman's correlation analysis of follicular fluid metabolites. Spearman's correlation between the biological antioxidant potential (BAP) and metabolites in follicular fluids using Graphpad 8. Confidence interval 95\%. BAP, biological antioxidant potential

that is able to induce more reactive oxygen species from mitochondria. The increase in oxidative stress due to the accumulation of ROS drives a series of events that induce a mechanism by which the oocytes enter apoptosis and lose their functionality (Aitken, 2020). The increase in oxidative stress due to both the production of androgens and an accumulation of lipids in the follicular environment can be one of the causes of reduced fertility. The small size of the sample is the main limitation of this study, future research using a large population and monitoring of long-term outcomes of IVF will be needed to confirm this preliminary information. Future investigations will also be focused on the morphology and metabolism of the cumulus cells and the follicle to confirm the changes observed in this study. 

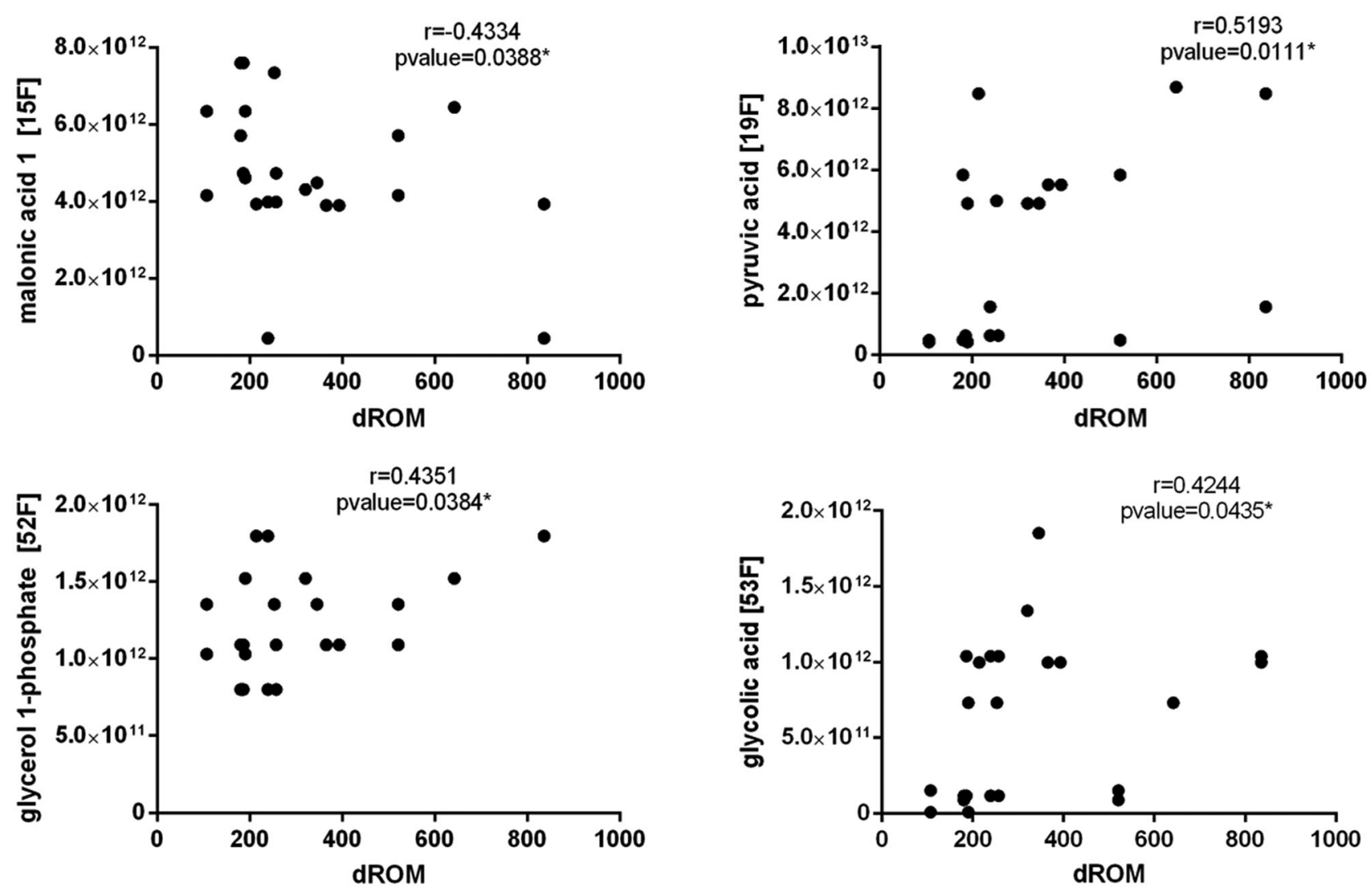

FIGURE 7 Spearman's correlation analysis of follicular fluid metabolites. Spearman's correlation between reactive oxygen metabolites (dROM) and metabolites in follicular fluids using Graphpad 8. Confidence interval 95\%. dROM, diacron reactive oxygen metabolites

\section{4 | MATERIALS AND METHODS}

\section{1 | Biological fluids collection}

Biological fluids were collected at the Centre for Diagnosis and Treatment of Couple Sterility, Medically Assisted Reproduction Unit, "Le Scotte" University Hospital in Siena. Thirty-one normo-ovulatory women, 30-42 years old, were recruited; all of them volunteered to participate in the study, and freely signed the informed consent. CEASVE local ethical committee approved the study.

\section{2 | Ovulation induction}

Controlled ovarian hyperstimulation was performed administering recombinant gonadotropins at a dose of 150-300 IU per day starting from Day 1st or 2nd of the spontaneous or induced menstrual cycle. The dose of gonadotropins was adjusted according to ovarian response, as monitorized by ovarian ultrasound examination and blood serum hormone levels evaluation.

When the dominant follicle reached the diameter of $14 \mathrm{~mm}$ a gonadotropin-releasing hormone antagonist was administered daily, until the day of ovulation triggering. As soon as at least three follicles reached $18 \mathrm{~mm}$ in diameter, the ovulation triggering was induced by recombinant human chorionic gonadotropin injection, and the pick- up was scheduled 34-36 hr after. The aspirated FFs were collected after oocyte recovery, centrifuged for $10 \mathrm{~min}$ at $1,500 \mathrm{rpm}$, and frozen at $-80^{\circ} \mathrm{C}$ until use. Oocyte maturity was evaluated and only FF corresponding to Metaphase 2 stage oocytes were selected. Moreover, blood samples were collected in the day of pick-up and the obtained sera were used for comparative analyses. Patients' characteristics are reported in Table 4.

\section{3 | Gas chromatography-MS (GC-MS) and sample treatment}

Serum and FF were subjected to metabolite analysis by GC-MS as reported by Lorito et al. (2020). Briefly, $200 \mu$ l of samples were supplemented with $200 \mu$ lice-cold MeOH (\#34854-1; Sigma Aldrich) and $200 \mu \mathrm{l}$ of chloroform (ratio 1:1:1; \#900688-1; Sigma Aldrich). Then samples were vortexed at $4^{\circ} \mathrm{C}$ for $20 \mathrm{~min}$, centrifuged at $3,000 \mathrm{~g}$ for $10 \mathrm{~min}$ at $4^{\circ} \mathrm{C}$ and the upper phase was collected in a new tube and evaporated at room temperature in a rotovapor. For derivatization, dried polar metabolites were dissolved in $60 \mu \mathrm{l}$ of $2 \%$ methoxyamine hydrochloride (\#226904; Sigma Aldrich) in pyridine (25104; Thermo Fisher Scientific) and held at $30^{\circ} \mathrm{C}$ for $90 \mathrm{~min}$. After dissolution and reaction, $90 \mathrm{ml}$ MSTFA + 1\% TMCS (69478-10x; Sigma Aldrich) was added, and samples were incubated at $37^{\circ} \mathrm{C}$ for $60 \mathrm{~min}$. Gas chromatographic runs were performed with helium as 
TABLE 4 Patients' characteristics

\begin{tabular}{|c|c|c|}
\hline Patient groups (age) & $\begin{array}{l}<35 \text { Between } 30 \\
\text { and } 35 \text { years old }\end{array}$ & $\begin{array}{l}>35 \text { Between } 39 \text { and } \\
44 \text { years old }\end{array}$ \\
\hline $\begin{array}{l}\text { Duration of } \\
\text { stimulation (days) }\end{array}$ & $10.5 \pm 0.9$ & $11.2 \pm 1.2$ \\
\hline No. of follicles & $6.5 \pm 1.5$ & $4.5 \pm 1.2$ \\
\hline No. of MII oocytes ${ }^{a}$ & $5.4 \pm 3.7$ & $3.5 \pm 1.8$ \\
\hline
\end{tabular}

${ }^{a}$ Oocytes reaching the metaphase II stage (MII) of maturation.

carrier gas at $0.6 \mathrm{ml} / \mathrm{min}$ at $250^{\circ} \mathrm{C}$ inlet temperature. The injection volume was $1 \mu \mathrm{l}$ and a split ratio of $1: 10$ was used. The GC oven temperature ramp was from $60^{\circ} \mathrm{C}$ to $325^{\circ} \mathrm{C}$ at $10^{\circ} \mathrm{C} / \mathrm{min}$. The data acquisition rate was $10 \mathrm{~Hz}$. For the Quadrupole, an El source $(70 \mathrm{eV})$ was used, and full-scan spectra (mass range from 50 to 600) were recorded in the positive ion mode.

The ion source and transfer line temperatures were set, respectively, to $250^{\circ} \mathrm{C}$ and $290^{\circ} \mathrm{C}$. The MassHunter data processing tool (Agilent) was used to obtain a global metabolic profiling using the Fihen Metabolomics RTL Library (G1676AA; Agilent). Statistical analysis was performed by GraphPad Prism 7.01.

\section{4 | Sample treatment and matrix-assisted laser desorption/ionization time-of-flight (MALDI-TOF) analysis}

Aliquots of $50 \mu \mathrm{l}$ of serum and FFs samples were used to perform lipid analysis. To each aliquot methanol $(200 \mu \mathrm{l})$ was added to precipitate the protein component. The samples were centrifuged for $10 \mathrm{~min}$ at 12,000 rpm (Glaser, Demmelmair, \& Koletzko, 2010; Zhang et al., 2020). The supernatant was filtered with a $0.45 \mu \mathrm{m}$ Millipore membrane filter (Merck, Darmstadt, Germany) and dried under vacuum. The samples were then suspended in $50 \mu$ of methanol containing $0.1 \%$ formic acid.

All of the extracts both from serum and FF $(0.5 \mu \mathrm{l})$ were deposited on MALDI plate with an equal volume (1/1, vol/vol) of a solution of 2,5-dihydroxybenzoic acid $(10 \mathrm{mg} / \mathrm{ml}$; Sigma Aldrich, Saint Louis) dissolved in 50:50 acetonitrile: $0.1 \%$ formic acid in water.

MALDI MS experiments were performed on a 4800 MALDITOF-TOF ABSciex equipped with a nitrogen laser $(337 \mathrm{~nm})$. Spectra were acquired in reflector positive mode by using a mass $(\mathrm{m} / \mathrm{z})$ range of 200-2,000 Da for lipids identification. Laser power was set to $4200 \mathrm{~V}$ for MS spectra acquisition. Each spectrum represents the sum of 3,000 laser pulses from randomly chosen spots per sample position by covering the entire spot. Calibration was performed by using $A B$ SCIEX calibration mixture (Monoisotopic $[M+n H] n+$ : 904.46 Da des-Arg-Bradykinin, 1296.68 Da Angiotensin I, 1570.67 Da Glu-Fibrinopeptide B, 2093.08 Da ACTH (clip: 1-17), 2465.19 Da ACTH (clip: 18-39), 3657.92 Da ACTH (clip: 7-38). The data were elaborated using the DataExplorer 5.1 software (Applied Biosystems, Foster City, CA) and the $\mathrm{m} / \mathrm{z}$ values were reported as monoisotopic masses. The MS/MS spectra were also recorded in positive mode for structural confirmation of identified lipids. For CID experiments, ambient air was used as collision gas with a medium pressure of 10-6 Torr. The Glu-Fibrinopeptide peptide (1570.67 Da) was used for the calibration of MS/MS acquisition.

\section{5 | Statistical analysis}

To evaluate the association between adiponectin and each parameter measured in FF and sera, nonparametric correlation Spearman test by GraphPad 8 was used. Significance was defined as $p<.05$.

\section{CONFLICT OF INTERESTS}

The authors declare that there are no conflict of interests.

\section{AUTHOR CONTRIBUTIONS}

S. L., T. F., and T. G. investigation, metabolomics, methodology, and visualization; F. M. resources; P. P., L. G., and A. L. provided biological samples; P. A. M. supervision and data curation; A. A., A. I., and G. P. MS analysis; A. M. writing, supervision, and data curation.

\section{DATA AVAILABILITY STATEMENT}

The data that support the findings of this study are available from the corresponding author upon reasonable request.

\section{ORCID}

Alessandra Modesti (D) http://orcid.org/0000-0002-1290-2285

\section{REFERENCES}

Aitken, R. J. (2020). Impact of oxidative stress on male and female germ cells: Implications for fertility. Reproduction, 159(4), R189-R201.

Berry, K. A., Hankin, J. A., Barkley, R. M., Spraggins, J. M., Caprioli, R. M., \& Murphy, R. C. (2011). MALDI imaging of lipid biochemistry in tissues by mass spectrometry. Chemical Reviews, 111(10), 6491-6512.

Boland, N. I., Humpherson, P. G., Leese, H. J., \& Gosden, R. G. (1993). Pattern of lactate production and steroidogenesis during growth and maturation of mouse ovarian follicles in vitro. Biology of Reproduction, 48, 798-806.

Bracewell-Milnes, T., Saso, S., Abdalla, H., Nikolau, D., Norman-Taylor, J., Johnson, M., ... Thum, M. Y. (2017). Metabolomics as a tool to identify biomarkers to predict and improve outcomes in reproductive medicine: A systematic review. Hum Reprod Update, 23(6), 723-736.

Chen, M., Zhang, B., Cai, S., Zeng, X., Ye, Q., Mao, X., ... Qiao, S. (2019). Metabolic disorder of amino acids, fatty acids and purines reflects the decreases in oocyte quality and potential in sows. J Proteomics, 30(200), 134-143.

Cordeiro, F. B., Montani, D. A., Pilau, E. J., Gozzo, F. C., Fraietta, R., \& Turco, E. G. L. (2018). Ovarian environment aging: Follicular fluid lipidomic and related metabolic pathways. Journal of Assisted Reproduction and Genetics, 35, 1385-1393.

Dludla, P. V., Nkambule, B. B., Jack, B., Mkandla, Z., Mutize, T., Silvestri, S., ... Mazibuko-Mbeje, S. E. (2018). Inflammation and oxidative stress in an obese state and the protective effects of gallic acid. Nutrients, 11(1), 23.

Freitas, C., Neto, A. C., Matos, L., Silva, E., Ribeiro, Â., Silva-Carvalho, J. L., \& Almeida, H. (2017). Follicular fluid redox involvement for ovarian follicle growth. Journal of Ovarian Research, 10(1), 44.

Gervais, A., Battista, M. C., Carranza-Mamane, B., Lavoie, H. B., \& Baillargeon, J. P. (2015). Follicular fluid concentrations of lipids and 
their metabolites are associated with intraovarian gonadotropinstimulated androgen production in women undergoing in vitro fertilization. J Clin Endocrinol Metab, 100(5), 1845-1854.

Glaser, C., Demmelmair, H., \& Koletzko, B. (2010). High-throughput analysis of fatty acid composition of plasma glycerophospholipids. Journal of Lipid Research, 51(1), 216-221.

Gonzalez, M. B., Lane, M., Knight, E. J., \& Robker, R. L. (2018). Inflammatory markers in human follicular fluid correlate with lipid levels and body mass index. J Reprod Immunol, 130, 25-29.

Gull, I., Geva, E., Lerner-Geva, L., Lessing, J. B., Wolman, I., \& Amit, A. (1999). Anaerobic glycolysis: The metabolism of the preovulatory human oocyte. European Journal of Obstetrics and Gynecology, 85, 225-228.

Harlow, C. R., Winston, R. M. L., Margara, R. A., \& Hillier, S. G. (2010). Gonadotropic control of human granulosa cell-glycolysis. Human Reproduction, 25, 949-956.

Jungheim, E. S., Macones, G. A., Odem, R. R., Patterson, B. W., Lanzendorf, S. E., Ratts, V. S., \& Moley, K. H. (2011). Associations between free fatty acids, cumulus oocyte complex morphology and ovarian function during in vitro fertilization. Fertility and Sterility, 95, 1970-1974.

Karnovsky, A., Weymouth, T., Hull, T., Tarcea, V. G., Scardoni, G., Laudanna, C., ... Omenn, G. S. (2012). Metscape 2 bioinformatics tool for the analysis and visualization of metabolomics and gene expression data. Bioinformatics, 28(3), 373-380.

Leopold, J., Popkova, Y., Engel, K. M., \& Schiller, J. (2018). Recent developments of useful MALDI matrices for the mass spectrometric characterization of lipids. Biomolecules, 8(4), 173.

Liu, X., \& Locasale, J. W. (2017). Metabolomics: A primer. Trends in Biochemical Sciences, 42(4), 274-284.

Lorito, N., Bacci, M., Smiriglia, A., Mannelli, M., Parri, M., Comito, G., ... Morandi, A. (2020). Glucose metabolic reprogramming of ER breast cancer in acquired resistance to the CDK4/6 inhibitor palbociclib. Cells, 9(3), 668.

Luti, S, Fiaschi, T, Magherini, F, Modesti, PA, Piomboni, P, Semplici, B, ... Gamberi, T. Follicular Microenvironment: Oxidative stress and adiponectin correlated with steroids hormones in women undergoing in vitro fertilization. Molecular Reproduction and Development. Accepted for publication.

Ma, J., Karnovsky, A., Afshinnia, F., Wigginton, J., Rader, D. J., Natarajan, L., ... Pennathur, S. (2019). Differential network enrichment analysis reveals novel lipid pathways in chronic kidney disease. Bioinformatics, 35(18), 3441-3452.

Ma, H., Sorokin, A., Mazein, A., Selkov, A., Selkov, E., Demin, O., \& Goryanin, I. (2007). The Edinburgh human metabolic network reconstruction and its functional analysis. Molecular Systems Biology, 3, 135.

Mai, K., Bobbert, T., Reinecke, F., Andres, J., Maser-Gluth, C., Wudy, S. A., ... Spranger, J. (2008). Intravenous lipid and heparin infusion-induced elevation in free fatty acids and triglycerides modifies circulating androgen levels in women: A randomized, controlled trial. Journal of Clinical Endocrinology and Metabolism, 93, 3900-3906.

Montani, D. A., Braga, D. P. A. F., Borges, E., Jr, Camargo, M., Cordeiro, F. B., Pilau, E. J., ... Lo Turco, E. G. (2019). Understanding mechanisms of oocyte development by follicular fluid lipidomics. Journal of Assisted Reproduction and Genetics, 36(5), 1003-1011.

Niringiyumukiza, J. D., Cai, H., \& Xiang, W. (2018). Prostaglandin E2 involvement in mammalian female fertility: Ovulation, fertilization, embryo development and early implantation. Reproductive Biology and Endocrinology, 16(1), 43.

Olszak-Wąsik, K., Bednarska-Czerwińska, A., Olejek, A., \& Tukiendorf, A. (2019). From "Every Day" hormonal to oxidative stress biomarkers in blood and follicular fluid, to embryo quality and pregnancy success? Oxidative Medicine and Cellular Longevity, 2019, 1092415.

Piñero-Sagredo, E., Nunes, S., de Los Santos, M. J., Celda, B., \& Esteve, V. (2010). NMR metabolic profile of human follicular fluid. NMR in Biomedicine, 23(5), 485-495.

Sneeringer, R., Penzias, A. S., Barrett, B., \& Usheva, A. (2011). High levels of mineralocorticoids in preovulatory follicular fluid could contribute to oocyte development. Fertility and Sterility, 95(1), 182-187.

Sutton-McDowall, M. L., Gilchrist, R. B., \& Thompson, J. G. (2010). The pivotal role of glucose metabolism in determining oocyte developmental competence. Reproduction, 139, 685-695.

Wu, L. L., Dunning, K. R., Yang, X., Russell, D. L., Lane, M., Norman, R. J., \& Robker, R. L. (2010). High-fat diet causes lipotoxicity responses in cumulus-oocyte complexes and decreased fertilization rates. Endocrinology, 151, 5438-5445A.

Xia, L., Zhao, X., Sun, Y., Hong, Y., Gao, Y., \& Hu, S. (2014). Metabolomic profiling of human follicular fluid from patients with repeated failure of in vitro fertilization using gas chromatography/mass spectrometry. International Journal of Clinical and Experimental Pathology, 7(10), 7220-7229.

Zhang, A. H., Ma, Z. M., Kong, L., Gao, H. L., Sun, H., Wang, X. Q., ... Wang, X. J. (2020). High-throughput lipidomics analysis to discover lipid biomarkers and profiles as potential targets for evaluating efficacy of Kai-Xin-San against APP/PS1 transgenic mice based on UPLC-Q/TOF-MS. Biomedical Chromatography, 34(2), e4724. https:// doi.org/10.1002/bmc.4724

Zhang, C. M., Zhao, Y., Li, R., Yu, Y., Yan, L. Y., Li, L., ... Qiao, J. (2014). Metabolic heterogeneity of follicular amino acids in polycystic ovary syndrome is affected by obesity and related to pregnancy outcome. BMC Pregnancy and Childbirth, 14, 11.

Zhao, Y., Fu, L., Li, R., Wang, L. N., Yang, Y., Liu, N. N., ... Qiao, J. (2012). Metabolic profiles characterizing different phenotypes of polycystic ovary syndrome: Plasma metabolomics analysis. BMC Medicine, 10, 153.

\section{SUPPORTING INFORMATION}

Additional supporting information may be found online in the Supporting Information section.

How to cite this article: Luti S, Fiaschi T, Magherini F, et al. Relationship between the metabolic and lipid profile in follicular fluid of women undergoing in vitro fertilization. Mol Reprod Dev. 2020;1-12. https://doi.org/10.1002/mrd.23415 